\title{
Transfer function based equivalent modeling method for wind farm
}

\author{
Feng WU ${ }^{1}$, Junxia QIAN ${ }^{1}$, Ping JU ${ }^{1}$, Xiaoping ZHANG ${ }^{2}$, \\ Yuqing JIN ${ }^{1}$, Dan XU ${ }^{1}$, Michael STERLING ${ }^{2}$
}

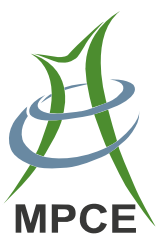

\begin{abstract}
To effectively study the dynamics of power systems with large-scale wind farms (WFs), an equivalent model needs to be developed. It is well known that back-toback converters and their controllers are important for the dynamic responses of the wind turbine (WT) under disturbances. However, the detailed structure and parameters of the back-to-back converters and their controllers are usually unknown to power grid operators. Hence, it is difficult to build an accurate equivalent model for the WF using the component model-based equivalent modeling method. In this paper, a transfer function based equivalent
\end{abstract}

CrossCheck date: 12 March 2018

Received: 28 July 2017 / Accepted: 12 March 2018/Published online: 7 May 2018

(C) The Author(s) 2018

$\triangle$ Feng WU

wufeng@hhu.edu.cn

Junxia QIAN

qianjunxia@jspdi.com.cn

Ping JU

pju@hhu.edu.cn

Xiaoping ZHANG

x.p.zhang@bham.ac.uk

Yuqing JIN

jyq16@hhu.edu.cn

Dan XU

1303859514@qq.com

Michael STERLING

mike@sterlingfamily.co.uk

1 College of Energy and Electrical Engineering, Hohai University, Nanjing 210098, China

2 Department of Electronic, Electrical and Computer Engineering, University of Birmingham, Birmingham B15 2TT, UK modeling method for the WF is proposed. During modeling, the detailed structure and parameters of the WF are not required. The objective of the method is reproducing the output dynamics of the WF under the variation of the wind speed and power grid faults. A decoupled parameter-estimation strategy is also developed to estimate the parameters of the equivalent model. A WF that consists of $16 \mathrm{WTs}$ is used to test the proposed equivalent model. Additionally, the proposed equivalent modeling method is applied to build the equivalent model for a real WF in Northwest China. The effectiveness of the proposed method is validated by the real measurement data.

Keywords Wind farm, Equivalent modeling, Transfer function, Parameter estimation, Particle swarm optimization

\section{Introduction}

Wind energy generation is developing very quickly. Many large-scale wind farms (WFs) have been integrated into the power grid, and they have had significant impact on the operation of the power grid [1]. The model of the $\mathrm{WF}$ is a major factor in evaluation; however, it has been widely recognized that it is inefficient to model each wind turbine (WT) in the WF in the dynamic simulation of the power grid integrated with large scale WFs. Hence, the equivalent model of the WF needs to be built to improve the efficiency of the simulation [2].

Many studies have been carried out to build the equivalent model for the WF. A one-machine equivalent model was developed in $[3,4]$ to represent the WF. Simulations showed that the dynamic responses of the aggregated model were very close to those of the detailed model under 
the disturbances of wind speed change and grid fault and the computational complexity was reduced significantly, but the impact factors were not discussed. In [5], the parameter calibration was performed when WF was large, with variations in the wind speed received by the individual WTs, the type of WTs installed in the WF, and the impedance of the collector system. After calibration, the accuracy of the one-machine model was improved under different conditions. In [6], 150 WTs in an offshore WF were separated into three equal groups, and a three-machine equivalent model was proposed to represent WF. The WT cluster method is very simple, and it is also difficult to carry out the parameter estimation for three machines. For the equivalent models mentioned above, the details of the WTs, collector network, and control system must be known before the equivalence, which is very difficult for power system operators.

The recently developed WTs, such as the WT with the doubly fed induction generator (DFIG) or direct drive permanent magnet generator, are interfaced to the power grid through the back-to-back converters, which decouple the inertia dynamics of the WT from the power grid. Hence, from the power grid's perspective, the dynamics of the WF are different from those of the traditional power plants, which are normally connected to the power grid directly. The converters and their controllers play important roles in the dynamic responses of the WTs under the conditions of power system faults and wind speed changes. For the installed WTs, it is difficult for the power grid operators to know the details of the WF, especially the structure of the controllers and their parameters. In this condition, the traditional equivalent modeling method based on the component models of the WT is not suitable to aggregate the dynamic models of the WF. Hence, it is necessary to study the equivalent modeling method for the WF [7].

The transfer function based equivalent modeling method has been widely used in equivalent modeling for power systems. This model has been successfully used to aggregate the dynamics of the electrical load and excitation system of the synchronous generator in [8] and [9], respectively. When using the transfer function to build the equivalent model, the object is regarded as a "black box" or a "grey box." The dynamics of the input and output of the object are used to build the equivalent model, while the details of its structure and parameters are not required. Hence, the transfer function based equivalent modeling method is suitable to be used to aggregate the dynamics of the WF.

In this paper, a transfer function based equivalent model is proposed to represent the dynamics of the WF under the power grid fault and wind speed variation. The coherence of the WTs in the WF are discussed to examine whether the conditions of the aggregation are satisfied. A decoupled parameter-estimation strategy is also developed, and particle-swarm optimization (PSO) is applied to estimate the parameters of the equivalent model of the WF. A WF consisting of 16 WTs with DFIG is built in MATLAB, and simulations are performed to test the proposed transfer function based equivalent model. The field-measured dynamics are also used to build the equivalent model for a real WF. The proposed equivalent model is hence validated.

\section{Transfer function based equivalent model for WF}

The output power of the WF changes under power system faults and variation of the wind speed. During a system fault, the terminal voltage drops sharply, and the output power of the WF changes quickly, which mainly depends on the control capability of the back-to-back converters and their controllers; when the wind speed varies, the output power of the WF changes with a short time delay because of the inertia of the WTs.

The equivalent model is built to describe the dynamics of the WF under both the system fault and wind speed variation. Because the output power of the WF changes quickly with the terminal voltage, the WF is regarded as a nonlinear equivalent impedance. Hence, the output power is proportional to the square of the terminal voltage. According to the aerodynamics of the WT, the output power is proportional to the cube of the wind speed. Hence, taking the terminal voltage and wind speed as the input, and the active power and reactive power of the WF as the output of the equivalent model, the transfer function based equivalent model for the WF is written as:

$$
\left\{\begin{array}{l}
P=P_{0}\left[H_{p u}(s)\left(\frac{U}{U_{0}}\right)^{2}\right]\left[H_{p v}(s)\left(\frac{v}{v_{0}}\right)^{3}\right] \\
Q=Q_{0}\left[H_{q u}(s)\left(\frac{U}{U_{0}}\right)^{2}\right]\left[H_{q v}(s)\left(\frac{v}{v_{0}}\right)^{3}\right]
\end{array}\right.
$$

where $P$ and $Q$ are the output active power and reactive power of the WF, respectively; $U$ and $U_{0}$ are the terminal voltage of the WF and its initial value, respectively; $v$ and $v_{0}$ are the input wind speed and its initial value, respectively; $H_{p u}, H_{p v}, H_{q u}$ and $H_{q v}$ are the transfer functions, and the uniform of the transfer functions can be written as follows: 
$H(s)=\frac{b_{m} s^{m}+b_{m-1} s^{m-1}+\cdots+b_{0}}{s^{n}+a_{n-1} s^{n-1}+\cdots+a_{0}}$

where $b_{m}, b_{m-1}, \ldots, b_{0}, a_{n-1}, a_{n-2}, \ldots, a_{0}$ are the constant coefficients; $m \leq n ; a_{0}=b_{0}$.

The configuration of the active power model is shown in Fig. 1, and the configuration of the reactive power model is similar to that of the active power model.

Compared to the traditional component-based equivalent modeling method, the transfer function modeling method treats the WF as a black box, and the equivalent model is an input/output (I/O) model, which does not have any explicit physical significance. Because the parameters of the WT in the WF are not required during the transfer function based equivalent modeling, its accuracy is lower than that of the model built by the component-based equivalent modeling method. Other similar methods, such as artificial neural network (ANN), can also be used to build the equivalent model for the WF. However, the ANN-based equivalent modeling method is difficult to be integrated into the commercial power system analysis software, whereas the transfer function based equivalent modeling method can be easily integrated into the commercial power system analysis software by the user definition function, including PSASP developed by China Electric Power Research Institute.

\section{Coherence of WTs in WF}

To aggregate the WTs in the WF into an equivalent model, the WTs in the WF must be coherent with each other [10]. For the synchronous generator, if the oscillations of the power angles between generators are coherent, these synchronous generators are coherent with each other, and can be aggregated into one equivalent synchronous generator. Meanwhile, the induction generators are usually used in the WTs and their inertias are decoupled by the power electronic devices. The power angle is no longer a suitable variable to determine the coherence of the WTs in the WF. However, the objective of the equivalent modeling is to minimize the error between the output power dynamics of equivalent model and the measurements. Under this condition, the dynamics of the output active

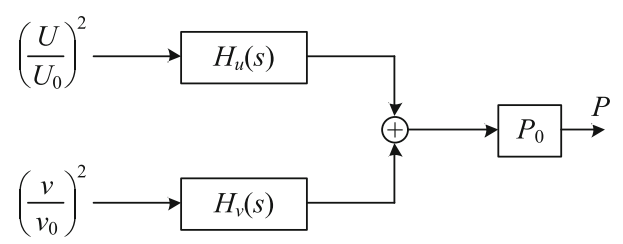

Fig. 1 Configuration of the equivalent model for active power power under the disturbance are used to study the coherence between the WTs in the WF.

A WF consisting of 16 WTs and interfaced into an infinite bus system via double transmission lines is built in MATLAB. The parameters of the simulation system are listed in the Appendix A. The configuration of the system is shown in Fig. 2. It is assumed that the wind flows into the WF from the left side of the WF. Considering the wake effect in the WF, the wind speeds flowing into different WTs are listed in Table 1. Simulations are carried out to obtain the dynamics of the output active power of the WTs under the system fault condition. Some of the dynamic responses of the WTs in the WF are shown in Fig. 3, and the dynamics of the WT under the system fault are close to each other. Applying the cluster analysis, the similarities among the different dynamic responses are analyzed using (3).

$$
r_{i j}=1-\frac{\sum\left(P_{i}(t)-P_{i}\left(t_{0}\right)\right)\left(P_{j}(t)-P_{j}\left(t_{0}\right)\right)}{\sqrt{\sum\left(P_{i}(t)-P_{i}\left(t_{0}\right)\right)^{2}} \sqrt{\sum\left(P_{j}(t)-P_{j}\left(t_{0}\right)\right)^{2}}}
$$

where $P_{i}$ and $P_{j}$ are the output active power of the $i$ th and $j$ th WT, respectively; $t$ and $t_{0}$ are the time of the sampling and its initial value; $r_{i j}$ is the similarity index, which demonstrates the difference between dynamics responses of the $i$ th WT and the $j$ th one.

If $r_{i j}$ is close to 0 , these dynamic responses are similar, and the $i$ th WT and $j$ th WT are coherent with each other. The results of the cluster analysis shown in Fig. 4 illustrate that $r_{i j}$ between the dynamic responses of different WTs in the WF are all lower than 0.01. Therefore, the WTs in the $\mathrm{WF}$ are coherent and can be aggregated to an equivalent model.

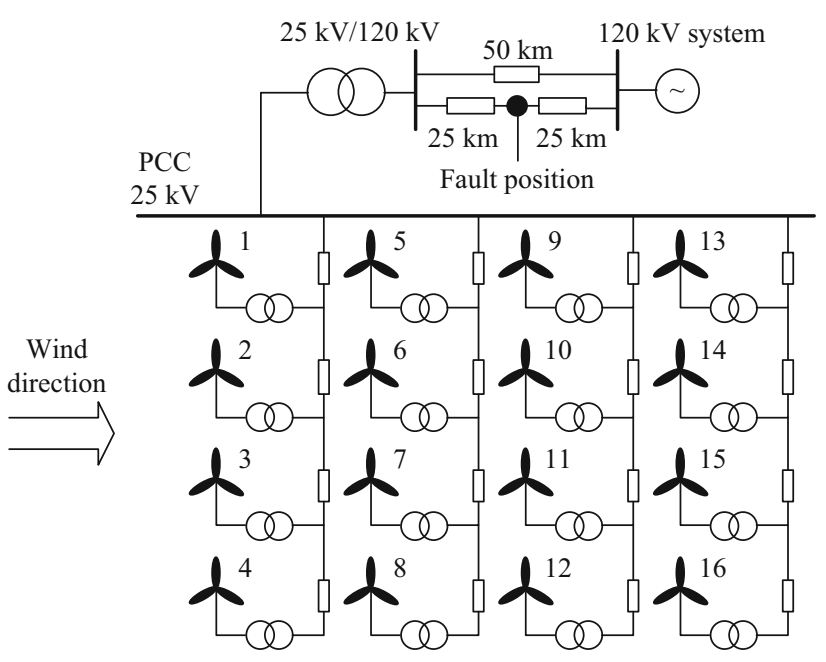

Fig. 2 Configuration of simulation system 
Table 1 Wind speeds of different WTs

\begin{tabular}{llrl}
\hline No. & Wind speed $(\mathrm{m} / \mathrm{s})$ & No. & Wind speed $(\mathrm{m} / \mathrm{s})$ \\
\hline 1 & 15.0 & 9 & 14.4 \\
2 & 13.8 & 10 & 12.8 \\
3 & 12.6 & 11 & 11.6 \\
4 & 11.4 & 12 & 10.4 \\
5 & 14.5 & 13 & 13.5 \\
6 & 13.3 & 14 & 12.3 \\
7 & 12.1 & 15 & 11.1 \\
8 & 10.9 & 16 & 9.9 \\
\hline
\end{tabular}

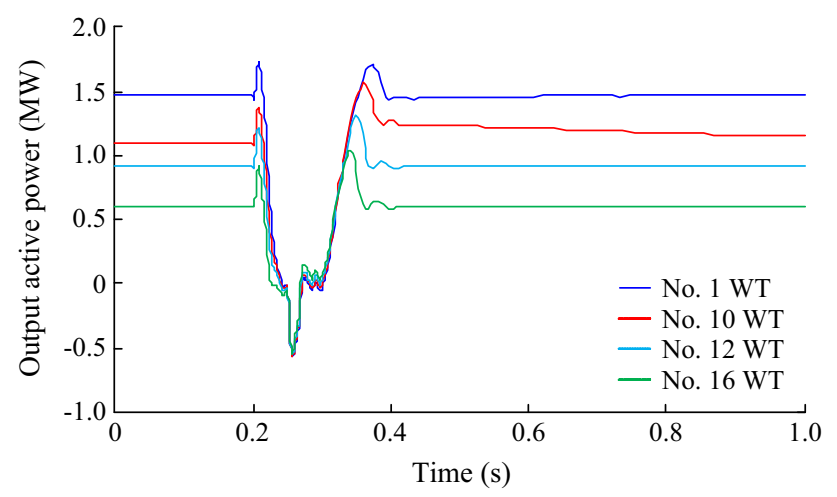

Fig. 3 Dynamic responses of different WTs

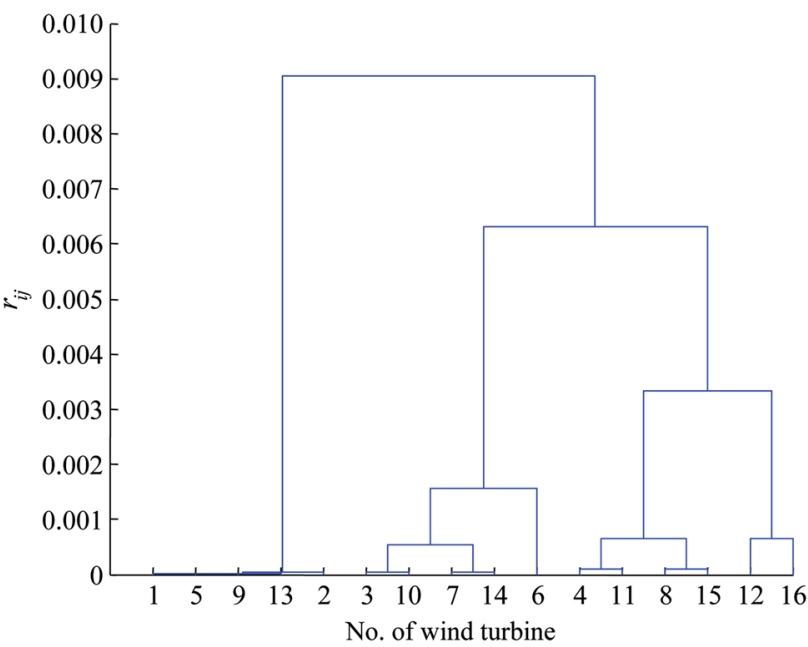

Fig. 4 Result of cluster analysis

\section{Decoupling parameter-estimation strategy for equivalent model}

The parameters of the transfer function based equivalent model include the parameters $H_{p u}(s), H_{p v}(s), H_{q u}(s)$ and $H_{q v}(s)$. There are tens of parameters in the equivalent model to be estimated. If all these parameters are estimated simultaneously, it is difficult for the parameter-estimation method to converge. Hence, the decoupled parameter-estimation strategy must be developed to obtain the parameters of the equivalent model.

Fortunately, as mentioned in Section 2, the dynamics of the WF under the system fault are much faster than those under the variation of the wind speed. In other words, they are decoupled with each other between time scales. The decoupling parameter-estimation strategy is proposed as follows.

When the parameters of the voltage-related transfer function $H_{p u}(s)$ and $H_{q u}(s)$ are estimated, the wind speed is assumed to be constant; when the parameters of the wind speed-related transfer function $H_{p v}(s)$ and $H_{q v}(s)$ are estimated, the terminal voltage of the WF is assumed to be constant. Additionally, the active power-related transfer function and the reactive power-related transfer function are independent of each other because of the decoupling control strategy, and their parameters are estimated separately. Therefore, the number of simultaneously estimated parameters is decreased significantly, and the efficiency of the parameter estimation is improved.

The PSO is used in the parameter estimation, and its flow chart is shown in Fig. 5. The details of the steps of PSO are presented in $[11,12]$. The steps of the parameter estimation for the voltage-related transfer function $H_{p u}(s)$ in the active power model are taken as the example to be introduced as follows.

Step 1: Initialization. In this step, bounds for the parameters, initial particles, bounds for the velocities for position updating, and initial velocities are generated. Step 2: Evaluation. The fitness values are calculated to evaluate the particles using the following fitness function:

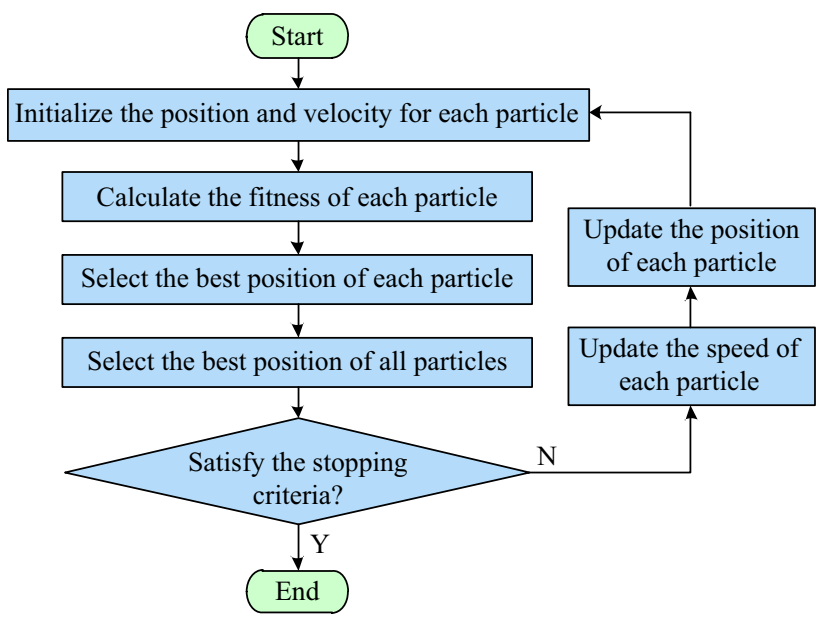

Fig. 5 Flow chart of PSO 
Table 2 Errors between simulated and calculated dynamics using voltage-related transfer-function model

\begin{tabular}{llll}
\hline Order of equivalent model & Number of parameters & Error of active power model (\%) & Error of reactive power model $(\%)$ \\
\hline 1 & 3 & 4.5906 & 23.8600 \\
2 & 5 & 2.3432 & 5.4816 \\
3 & 7 & 0.7124 & 1.8621 \\
4 & 9 & 0.7040 & 1.8206 \\
5 & 11 & 0.7032 & 1.8181 \\
\hline
\end{tabular}

Table 3 Parameters of $3^{\text {rd }}$-order voltage-related model

\begin{tabular}{lcrrl}
\hline Model & \multicolumn{1}{l}{$b_{3}$} & \multicolumn{1}{l}{$b_{2}$} & \multicolumn{1}{l}{$b_{1}$} & \multicolumn{1}{l}{$b_{0}$} \\
\hline$P-U$ & -1.5067 & 4.3238 & -5.7532 & 2.6132 \\
$Q-U$ & -6.8280 & 175.6800 & 4.2096 & 1.3083 \\
\hline Model & $a_{2}$ & & $a_{1}$ & $a_{0}$ \\
\hline$P-U$ & 36.0100 & 40.8100 & 2.6132 \\
$Q-U$ & 4.9014 & 0.6332 & 1.3083 \\
\hline
\end{tabular}

$F=\min \left\{\sum_{l=1}^{c_{\mathrm{c}}}\left(P_{l m}-P_{l c}\right)^{2}\right\}$

where $P_{l m}$ is the measured output active power of the WF; $P_{l c}$ is the calculated output active power of the WF equivalent model; $c_{\mathrm{c}}$ is the number of the sampling.

Step 3: Loop. Stopping criteria are checked. If the maximum number of iterations is reached or the fitness value is smaller than a specified value, the optimization stops, and the particle with the smallest fitness value is taken as the estimated parameter for the equivalent model; otherwise, it goes to Step 4.

Step 4: Updating velocities and positions. The detailed method for velocity and position updating can be found in $[12,13]$. After the update, go to Step 2.

\section{Effectiveness evaluation of equivalent modeling method}

\subsection{Model evaluated using the simulations in MATLAB}

1) Parameter estimation of the voltage-related transfer function

When the parameters of the voltage-related transfer function are estimated, the wind speed is assumed to be constant. Using the simulation system mentioned in Section 3 and applying a three-phase short-circuit fault at the middle of transmission line, as shown in Fig. 2, the
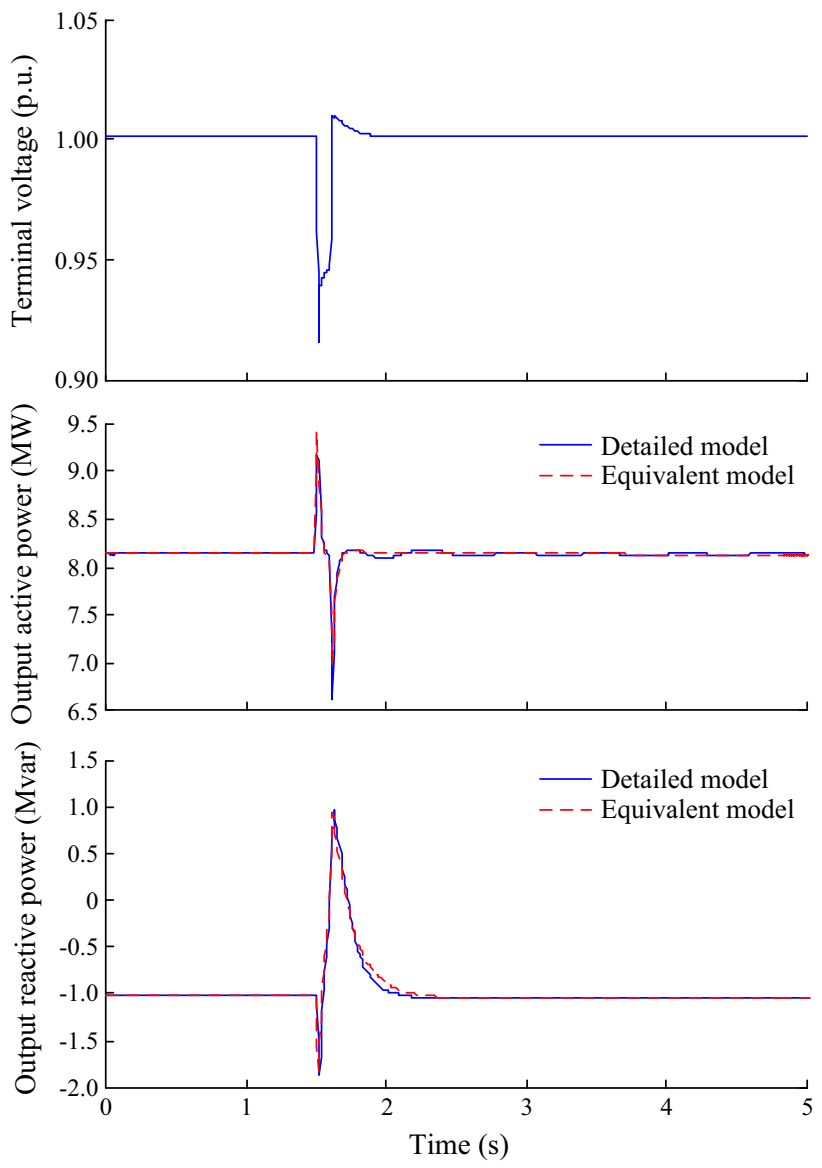

Fig. 6 Dynamics of $3^{\text {rd }}$-order voltage-related model

dynamics of the WF are simulated. Taking the simulated dynamics as the measurements of the WF, the parameters of the voltage-related transfer function with different orders are estimated. The errors between the simulated dynamics and the outputs of the equivalent models with different orders are listed in Table 2. From Table 2, it can be seen that the errors between the simulated dynamics and those of the outputs of the equivalent model decrease significantly from the $1^{\text {st }}$-order model to $3^{\text {rd }}$-order model, whereas the errors decrease slightly when the order of the transfer function is greater than 3 . Hence, the $3^{\text {rd }}$-order 
Table 4 Errors between simulated and calculated dynamics using voltage-related transfer-function model

\begin{tabular}{lccc}
\hline Order of equivalent model & Number of parameters & Error of active power model $(\%)$ & Error of reactive power model $(\%)$ \\
\hline 1 & 2 & 13.9235 & 34.4588 \\
2 & 4 & 7.4014 & 10.8986 \\
3 & 6 & 0.8102 & 3.7168 \\
4 & 8 & 0.7988 & 3.5921 \\
5 & 10 & 0.7884 & 3.5331 \\
\hline
\end{tabular}

Table 5 Parameters of $3^{\text {rd }}$-order wind speed-related model

\begin{tabular}{lllllll}
\hline Model & $b_{2}$ & $b_{1}$ & $b_{0}$ & $a_{2}$ & \multicolumn{1}{l}{$a_{1}$} & $a_{0}$ \\
\hline$P-U$ & 1.6826 & 4.1659 & 0.3545 & 19.5 & 10.4600 & 0.3545 \\
$Q-U$ & 7.4130 & 2.0827 & 0.0301 & 16.1 & 0.7027 & 0.0301 \\
\hline
\end{tabular}
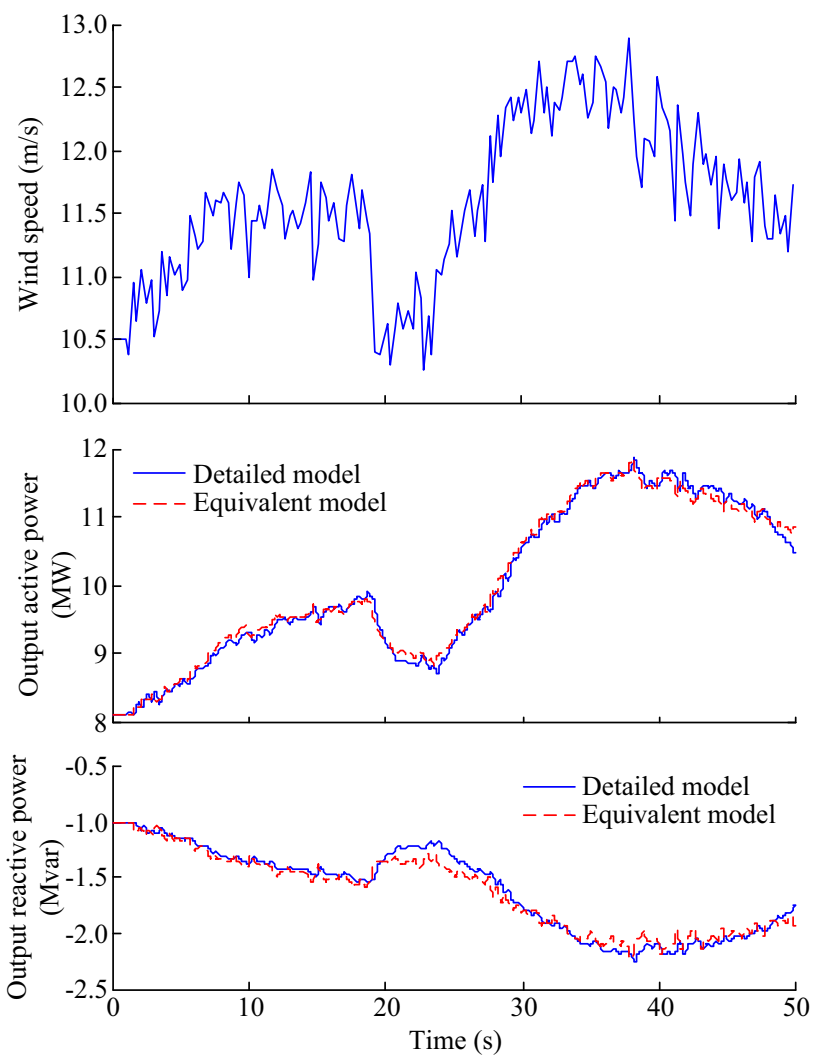

Fig. 7 Dynamics of WF under variation of wind speed

transfer function is the most cost-efficient model for the voltage-related equivalent model, and it is used in the further study in this paper. The estimated parameters of the $3^{\text {rd }}$-order model are listed in Table 3. The dynamics of the outputs of the $3^{\text {rd }}$-order model are also shown in Fig. 6 . The dynamics of the output of the $3^{\text {rd }}$-order model are very close to those simulated.
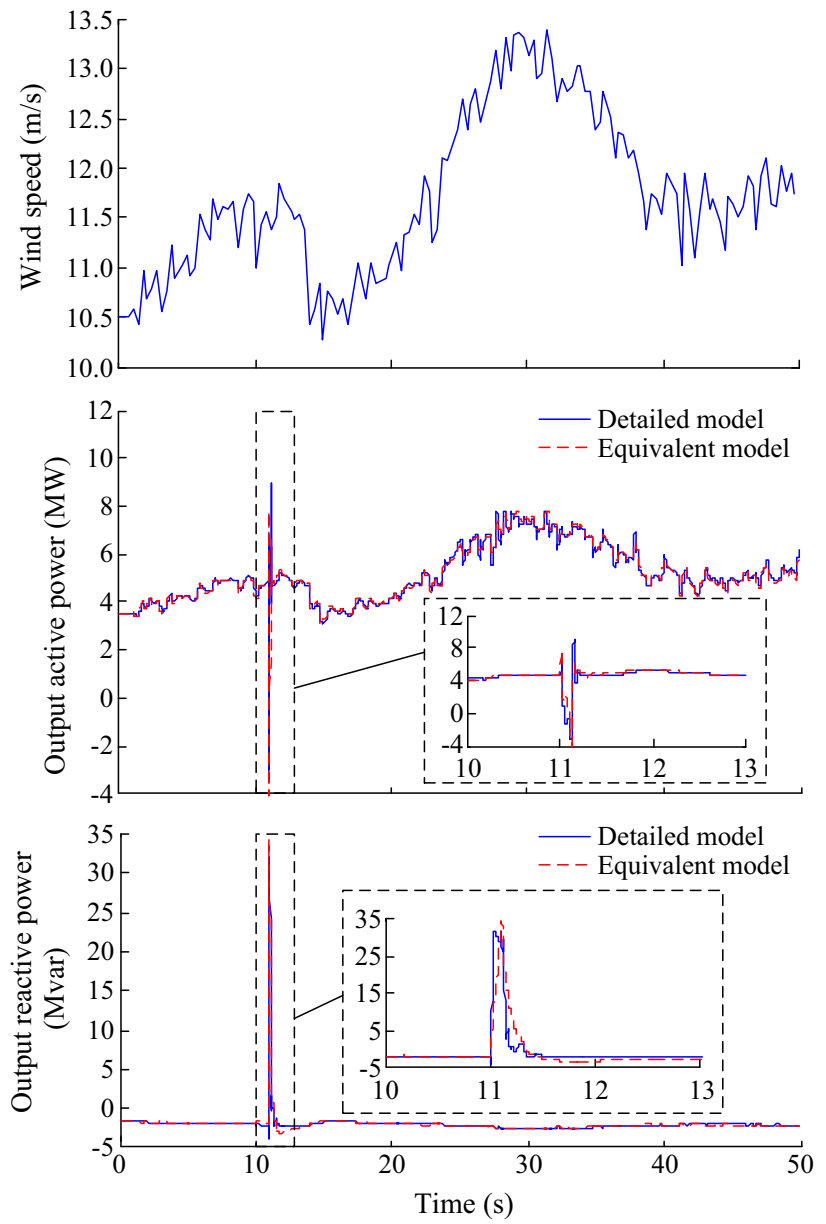

Fig. 8 Dynamics of equivalent model and detailed model

2) Parameter estimation of the wind speed-related transfer function

When the parameters of the wind speed-related transfer function are estimated, the terminal voltage of the WF is assumed to be constant. The wind speed, consisting of basic wind, gusty wind, ramp wind, and noisy wind, is the input variable of the WF, as shown in Fig. 2. Considering the wake effect, the wind speed decreases by $5 \%$ after flowing through a WT. The simulations are performed. The input wind speed and the output active and reactive power 


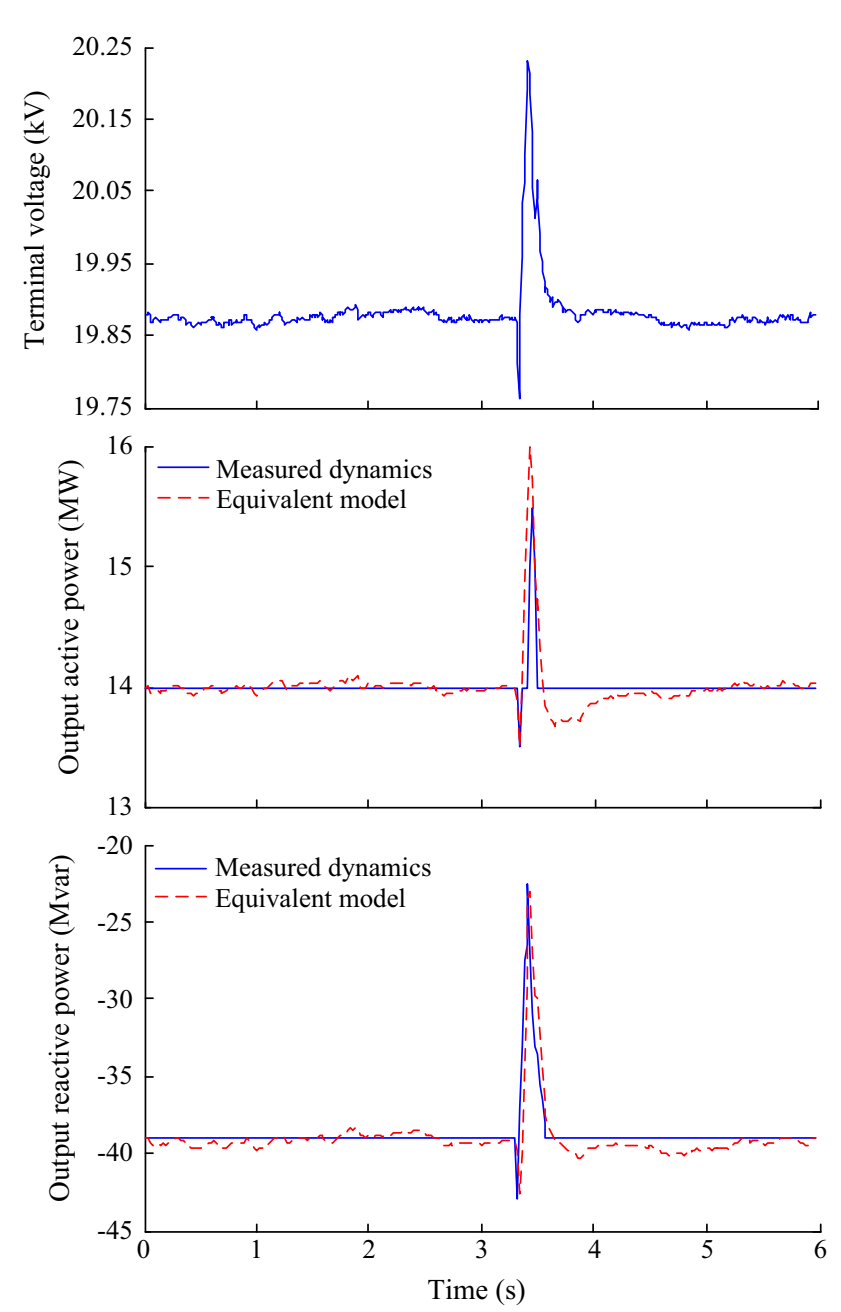

Fig. 9 Dynamics of recorded output power and those of equivalent model under system fault

of the WF are taken as the measurements to build the equivalent model.

Because the inertia of the WT is large and the dynamics of the output power are slightly delayed, $b_{m}$ in (2) of the wind speed-related transfer function is set to 0 . The parameters of the wind speed-related transfer function with different orders are estimated. The errors between the simulated dynamics and the dynamics of the equivalent model with different orders are also listed in Table 4. From Table 4 , it is concluded that the $3^{\text {rd }}$-order model is the most suitable one. Hence, the $3^{\text {rd }}$-order transfer function is used in the later works of this paper. The estimated parameters of the $3^{\text {rd }}$-order model are listed in Table 5, and the dynamics of the output active and reactive power of the $3^{\text {rd }}$-order model are also shown in Fig. 7. The dynamics of the output of the $3^{\text {rd }}$-order model and those simulated match very well.
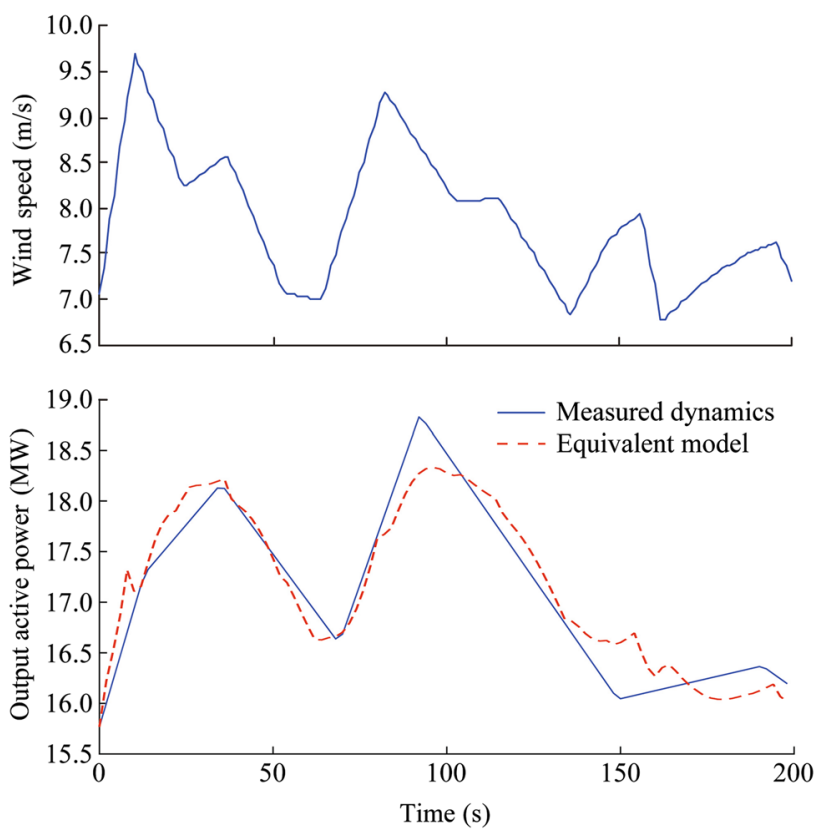

Fig. 10 Dynamics of recorded output power and those of equivalent model under wind speed variation

\section{3) Evaluation of the equivalent model}

Based on the voltage-related and wind speed-related model, the transfer function based equivalent model of the WF is built as follows:

$$
\left\{\begin{array}{c}
P=P_{0}\left[\frac{-1.5067 s^{3}+4.3238 s^{2}-5.7532 s+2.6132}{s^{3}+36.01 s^{2}+40.81 s+2.6132}\left(\frac{U}{U_{0}}\right)^{2}\right] \\
\cdot\left[\frac{1.6826 s^{2}+4.1659 s+0.3545}{s^{3}+19.5 s^{2}+10.46 s+0.3545}\left(\frac{v}{v_{0}}\right)^{3}\right] \\
Q=Q_{0}\left[\frac{-6.8280 s^{3}+175.68 s^{2}+4.2096 s+1.3083}{s^{3}+4.9014 s^{2}+0.6332 s+1.3083}\left(\frac{U}{U_{0}}\right)^{2}\right] \\
\cdot\left[\frac{7.4130 s^{2}+2.0827 s+0.0301}{s^{3}+16.1 s^{2}+0.7027 s+0.0301}\left(\frac{v}{v_{0}}\right)^{3}\right]
\end{array}\right.
$$

The model is built in MATLAB. To evaluate the robustness of the equivalent model, another wind speed shown in Fig. 8 is injected into the WF, and a three-phase short-circuit fault is applied to the middle of the transmission line at $11 \mathrm{~s}$ and cleared after $0.1 \mathrm{~s}$. The simulations are carried out, and the dynamics of output active and reactive power during the entire simulation period and around the time of the fault are shown in Fig. 7. The dynamics of the equivalent model are very close to those of the detailed WF model. The errors in the active power and reactive power are 0.82 and $4.20 \%$, respectively. 


\subsection{Model evaluation using field measurements}

The phasor measurement unit (PMU) is installed at the terminal of a WF, which is located in Northwest China. The WF comprises 20 WTs with DFIG. Because the capacity of each WT is $1.5 \mathrm{MW}$, the total capacity of the WF is $30 \mathrm{MW}$. A system fault happened on December 15, 2012, and the dynamics of the terminal voltage, output active power, and output reactive power are recorded and shown in Fig. 8. Using the recorded dynamics, the voltagerelated transfer function of the equivalent model of the WF is built as follows:

$$
\left\{\begin{array}{l}
P=P_{0}\left[\frac{-3.42 s^{3}+45.34 s^{2}+5.18 s+2.24}{s^{3}+38.37 s^{2}+25.23 s+2.24}\left(\frac{U}{U_{0}}\right)^{2}\right] \\
Q=Q_{0}\left[\frac{-14.32 s^{3}-273.92 s^{2}+14.18 s+4.24}{s^{3}+48.37 s^{2}+43.78 s+4.24}\left(\frac{U}{U_{0}}\right)^{2}\right]
\end{array}\right.
$$

The dynamics of the output active and reactive power of the equivalent model are also shown in Fig. 9. The output dynamics of the equivalent model are very close to the recorded dynamics. The errors between the measured dynamics and those of the equivalent model are 0.37 and $1.7 \%$ for active power and reactive power, respectively.

The measured dynamics of the output active power of the real WF under the variation of the wind speed is also chosen to build the wind speed-related equivalent model. The variations of the wind speed and active power are shown in Fig. 10. The equivalent model is built as follows:

$P=P_{0}\left[\frac{0.6023 s^{3}+0.0584 s^{2}+0.0301 s+0.0005}{s^{3}+0.7808 s^{2}+0.0514 s+0.0005}\left(\frac{v}{v_{0}}\right)^{3}\right]$

The active power of the equivalent model is also shown in Fig. 10. The output power of the equivalent model is close to the recorded value. The error between the measured dynamics and the output of the equivalent model is $2.86 \%$.

\section{Conclusion}

In this paper, an equivalent modeling method for the WF has been proposed. Because the equivalent model is based on the transfer function, the objective of the modeling is to describe the relation between the dynamics of the WF output and input variation. The detailed structure and parameters of the WF are not necessary, which is very convenient during the construction of the equivalent model. A decoupled parameter-estimation strategy has also been developed to estimate the parameters of the equivalent model, and the efficiency of the parameter estimation has been improved. The proposed equivalent modeling method has been employed to build the aggregated model for a simulation system and a real WF. The outputs of the equivalent models match well with those of the WF. The proposed equivalent model is confirmed to be effective to aggregate the dynamics of the WF using actual, real-time measurements.

Acknowledgment This work was supported by National Natural Science Foundation of China (No. 51422701). The authors would also like to thank the support of Chinese National "111" Project of "Renewable Energy and Smart Grid" at Hohai University.

Open Access This article is distributed under the terms of the Creative Commons Attribution 4.0 International License (http:// creativecommons.org/licenses/by/4.0/), which permits unrestricted use, distribution, and reproduction in any medium, provided you give appropriate credit to the original author(s) and the source, provide a link to the Creative Commons license, and indicate if changes were made.

\section{Appendix A}

Parameters of the simulation system are given as follows:

1) WT: $H_{t}=3 \mathrm{~s}, H_{g}=0.5 \mathrm{~s}, K_{s h}=10, D_{s h}=3.14$.

2) DFIG: $R_{s}=0.00706, L_{s}=0.171, L_{m}=2.9, \quad R_{r}=0.005$, $L_{r}=0.156, L_{s s}=L_{s}+L_{m}, L_{r r}=L_{r}+L_{m}$.

3) Converter: $C=0.001, V_{\mathrm{DC} 0}=1200 \mathrm{~V}, X_{T g}=0.55$.

4) Collection system: $R=0.1153 \Omega / \mathrm{km}, L=1.05 \times 10^{-3}$ $\mathrm{H} / \mathrm{km}, C=11.33 \times 10^{-9} \mathrm{~F} / \mathrm{km}, l=0.6 \mathrm{~km}$

5) Transmission line: $S_{N}=2500 \mathrm{MVA}, U_{N}=120 \mathrm{kV}, R=$ $0.1153 \Omega / \mathrm{km}, L=1.05 \times 10^{-3} \mathrm{H} / \mathrm{km}, C=11.33 \times 10^{-9}$ $\mathrm{F} / \mathrm{km}, l=50 \mathrm{~km}$.

\section{Reference}

[1] Wang L, Vo QS, Hsieh MH et al (2017) Transient stability analysis of Taiwan power system's power grid connected with a high-capacity offshore wind farm. In: Proceedings of 2017 international conference on system science and engineering (ICSSE), Ho Chi Minh City, Vietnam, 21-23 July 2017, pp 585-590

[2] Garcia CA, Fernandez LM, Jurado F (2015) Evaluating reduced models of aggregated different doubly fed induction generator wind turbines for transient stabilities studies. Wind Energy 18(1):133-152

[3] Fernandez LM, Garcia CA, Saenz JR et al (2006) Reduced model of dfigs wind farms using aggregation of wind turbines and equivalent wind. In: Proceedings of IEEE electrotechnical conference, Torremolinos, Spain, 16-19 May 2006, pp 881-884 
[4] Muljadi E, Ellis A (2008) Validation of wind power plant models. In: Proceedings of IEEE power and energy society general meeting - conversion and delivery of electrical energy in the 21st century, Pittsburgh, USA, 20-24 July 2008, 7 pp

[5] Elizondo MA, Lu S, Zhou N et al (2011) Model reduction, validation, and calibration of wind power plants for dynamic studies. In: Proceedings of IEEE power and energy society general meeting, San Diego, USA, 24-29 July 2011, 8 pp

[6] Turegano JM, Villalba SA, Herraiz GC et al (2017) Model aggregation of large wind farms for dynamic studies. In: Proceedings of $43^{\text {rd }}$ annual conference of the IEEE industrial electronics society, Beijing, China, 29 October-1 November 2017, pp 316-321

[7] Zou J, Peng C, Xu H et al (2015) A fuzzy clustering algorithmbased dynamic equivalent modeling method for wind farm with DFIG. IEEE Trans Energy Convers 30(4):1329-1337

[8] IEEE Task Force on Load Representation for Dynamic Performance (1995) Bibliography on load models for power flow and dynamic performance simulation. IEEE Trans Power Syst 10(1):523-538

[9] Zhou HQ, Huang XC, Wu L et al (2010) Aggregation of excitation systems based on standard transfer functions. Autom Electric Power Syst 34(1):15-19

[10] Sun T, Mou X, Li Z (2015) A practical clustering method of DFIG wind farms based on dynamic current error. In: Proceedings of IEEE power and energy society general meeting, Denver, USA, 26-30 July 2015, 5 pp

[11] Liu ZH, Wei HL, Zhong QC et al (2017) Parameter estimation for VSI-fed PMSM based on a dynamic PSO with learning strategies. IEEE Trans Power Electron 32(4):3154-3165

[12] Silva SAO, Sampaio LP, Oliveira FM et al (2017) Feed-forward DC-bus control loop applied to a single-phase grid-connected PV system operating with PSO-based MPPT technique and active power-line conditioning. IET Renew Power Gener 11(1):183-193

[13] Wu F, Zhang XP, Godfrey K et al (2007) Small signal analysis and optimal control of wind turbine with doubly fed induction generator. IET Gener Transm Distrib 1(5):751-760

Feng WU received the B.Eng. and M.Sc. degrees in Electrical Engineering from Hohai University, China, in 1998 and 2002, respectively, and received the Ph.D. degree also in Electrical Engineering from the University of Birmingham, UK in 2009. He is current a professor at Hohai University, China. His research interest is modeling and control of the renewable energy generation.

Junxia QIAN received the B.Eng. degree in Mechanical Engineering from Nantong University in 1999, and received M.Sc. degree in automation from Hohai University in 2005. She is currently pursuing the Ph.D. degree in Electrical Engineering at Hohai University. Her research interest is modeling of the renewable energy generation.
Ping JU received the B.Eng. and M.Sc. degrees in Electrical Engineering from Southeast University, China in 1982 and 1985, respectively. In 1988 he received the Ph.D. degree in Electrical Engineering from Zhejiang University, Hangzhou, China. He was an Alexander-von-Humboldt Fellow at the University of Dortmund, Germany. He is now a professor of electrical engineering in the College of Electrical Engineering at Hohai University, Nanjing, China. His research interest is modeling and control of the power system.

Xiaoping ZHANG received the B.Eng., M.Sc. and Ph.D. degrees in Electrical Engineering from Southeast University in 1988, 1990, 1993, respectively. He worked at NARI, Ministry of Electric Power, China on EMS/DMS advanced application software research and development from 1993 to 1998 . He visited UMIST from 1998 to 1999. He was an Alexander-von-Humboldt Research Fellow with the University of Dortmund, Germany from 1999 to 2000. He was a lecturer and then an associate professor at the University of Warwick, UK till early 2007. Currently he is a professor at the University of Birmingham, UK. His research interest is technologies for smart grids.

Yuqing JIN received the B.Eng., M.Sc. and Ph.D. degrees in Electrical Engineering from Hohai University in 2002, 2006, 2012, respectively. He is currently an associate professor at Hohai University. His research interest is modeling of the wind farm.

Dan XU received the B.Eng. and M.Sc. degrees in Electrical Engineering from Hohai University in 2014 and 2017, respectively. She is currently an electrical engineer at SUMEC Co. Ltd. Nanjing, China. Her research interest is modeling of the ocean energy generation.

Michael STERLING received a B.Eng. degree with First Class Honours in Electronic and Electrical Engineering at the University of Sheffield and subsequently a Ph.D. degree in computer control in 1971. In 1971 he joined the Department of Control Engineering, University of Sheffield, as a lecturer, being promoted to senior lecturer in 1978. In 1980 he was appointed as professor of engineering at the University of Durham, UK. In 1989 he was appointed vicechancellor and principal of Brunel University. He was vice-chancellor at the University of Birmingham. He is a fellow of the Institute of Measurement and Control, the Institution of Electrical Engineers (IET) and the Royal Academy of Engineering. He has served on the IET Council, and is a former chairman of the Russell Group, and is also a past president of the Institute of Electrical Engineers and the Institute of Measurement and Control. His research interest is operation of the power system. 